\title{
Case report and literature review of popliteal artery entrapment syndrome
}

This article was published in the following Dove Press journal:

International Journal of General Medicine

6 July 2015

Number of times this article has been viewed

\author{
Nedaa Skeik' \\ Taylor M Thomas ${ }^{2}$ \\ Bjorn I Engstrom ${ }^{3}$ \\ Jason Q Alexander ${ }^{4}$ \\ 'Vascular Medicine, Minneapolis Heart \\ Institute, ${ }^{2}$ Research, Minneapolis \\ Heart Institute Foundation, \\ ${ }^{3}$ Interventional Radiology, Minneapolis \\ Heart Institute, ${ }^{4}$ Vascular Surgery, \\ Minneapolis Heart Institute, MN, USA
}

\begin{abstract}
Popliteal artery entrapment syndrome (PAES) is an uncommon condition resulting from an abnormal anatomic relationship between the popliteal artery and the surrounding musculature. The compression created by this variance in anatomy can lead to ischemia and vascular claudication. The diagnosis of PAES requires a thorough patient history and physical exam, a high index of suspicion, and dedicated imaging techniques. Several treatment options are available, including surgical intervention, thrombolysis, or a combination of these depending on the clinical indication. We present a case of PAES in a 34-year-old man who presented with typical symptoms illustrative of the complicated decision making related to this disorder.
\end{abstract}

Keywords: popliteal artery thrombosis, limb ischemia, revascularization

\section{Case report}

A 34-year-old man presented with abrupt onset of intermittent exercise-induced pain in the left calf that improved with rest. He denied any trauma to the lower extremity. Past medical history was significant for 14 years of tobacco abuse, and 1-year use of electronic cigarette. He denied history or symptomatology concerning for vasculitis, autoimmune, connective tissue, thrombotic or rheumatologic disorders.

Physical examination revealed normal vital signs with palpable left femoral but absent popliteal, posterior tibial, and dorsalis pedis pulses. In spite of the absence of pulses on the left lower limb, the patient's foot did not have other findings of critical ischemia. The remainder of the physical exam was unremarkable.

Arterial duplex ultrasound showed a complete occlusion of the left popliteal artery. The patient was started on anticoagulation with enoxaparin to bridge to warfarin. Magnetic resonance angiogram of abdomen and pelvis with three-vessel runoff revealed widely patent common femoral, profunda femoral, and proximal-mid superficial femoral arteries. However, distal segment of the left superficial femoral and above-knee popliteal arteries showed abrupt occlusion, with the occluded segment spanning $9 \mathrm{~cm}$ (Figure 1). Mild medical deviation of bilateral above-knee popliteal artery was also present suggesting extrinsic compression laterally, although with patent right arterial system (Figure 2). This deviation was concluded to be related to the proximal and lateral aberrant insertion of the medial head of the gastrocnemius muscle in the left leg and due to a fibrous band or muscle slip in the right leg (Figures 1-3). Catheter-based angiogram was performed 3 days later and confirmed the diagnosis of distal superficial femoral and popliteal artery occlusion with possible popliteal entrapment syndrome as
Correspondence: Nedaa Skeik, Department of Vascular Medicine, Minneapolis Heart institute, 920 E 28th St, Minneapolis, MN 55407, USA

Email Nedaa.skeik@allina.com 


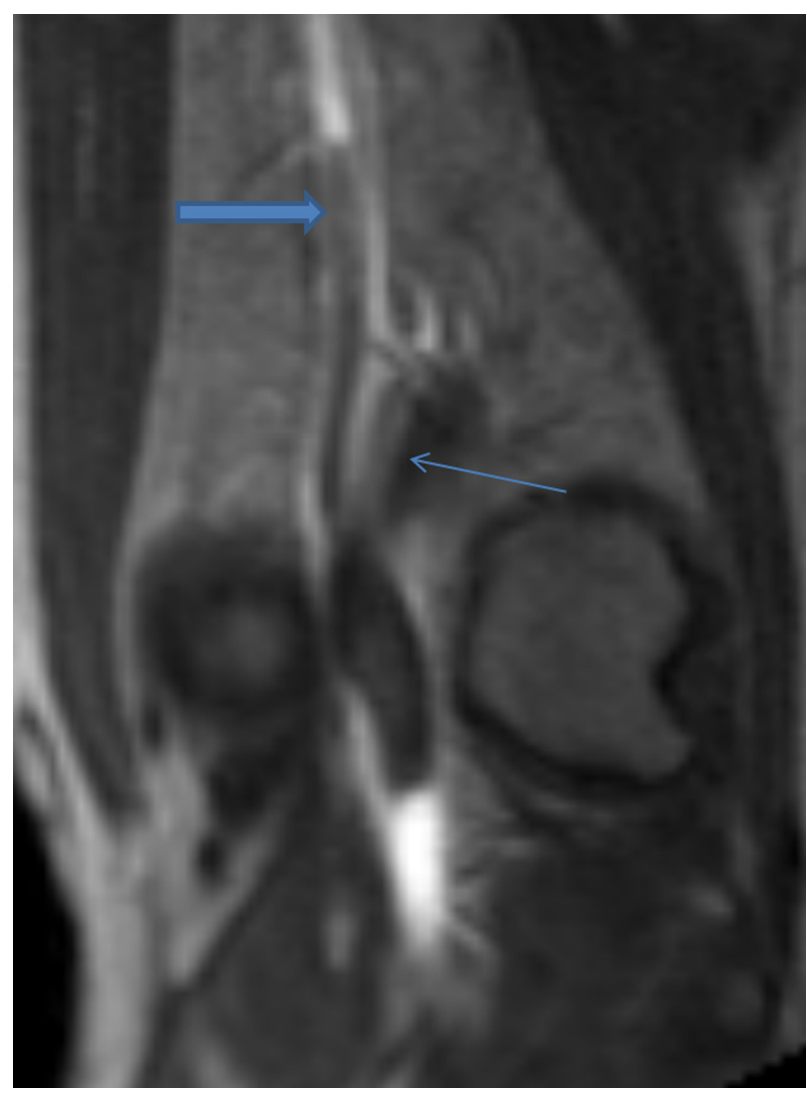

Figure I Fast low-angle shot 3D coronal TI-weighted post-contrast magnetic resonance image of the left knee demonstrates occlusion of the above-knee popliteal artery (thick arrow) as well as a lateral aberrant insertion of the medial head of the gastrocnemius muscle onto the distal femur (thin arrow).

the etiology (Figure 4). It also revealed significant infrapopliteal artery disease suggestive of thromboembolic event versus Buerger's disease (Figure 5).

Management was started with enoxaparin $(80 \mathrm{mg})$ subcutaneous injection twice daily, aspirin $(81 \mathrm{mg})$ once daily and Lipitor $(40 \mathrm{mg})$ at night. Due to the severity of his symptoms, the patient underwent catheter-based lytic therapy using tissue plasminogen activator at $1 \mathrm{mg} / \mathrm{h}$ delivered through a multi-sidehole infusion catheter with heparin at 500 units/h through the sidearm of the sheath over 48 hours (Figure 6), followed by left lower extremity superficial femoral artery-to-below-knee popliteal artery bypass using ipsilateral reverse saphenous vein graft tunneled beneath the sartorius and below-knee popliteal artery endarterectomy, with good results. Following surgery, the patient was continued on warfarin to complete at least 3 months of anticoagulation. A repeat duplex arterial ultrasound was performed 2 weeks post-surgery and showed a widely patent distal superficial femoral artery to below-knee popliteal vein bypass graft without evidence of stenosis.

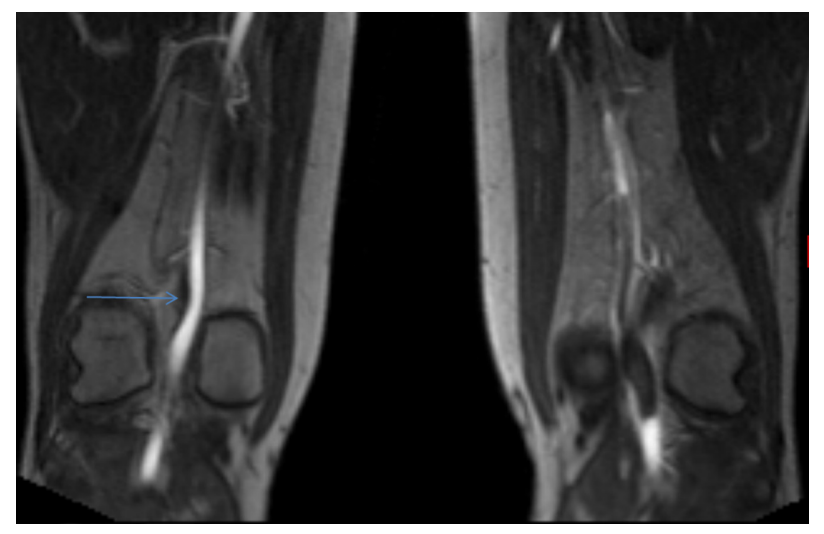

Figure 2 Flash 3D coronal TI-weighted post-contrast magnetic resonance image of bilateral knees.

Note: The arrow indicates a thin fibrous band of small muscle slip on the contralateral side with mild medial deviation of the popliteal artery (the asymptomatic side).

\section{Discussion}

Popliteal artery entrapment syndrome (PAES) is an important but infrequent condition that occurs as a result of compression of the popliteal artery by adjacent muscles and tendons, ${ }^{1}$ often resulting in ischemia and vascular claudication. ${ }^{2}$ Most commonly seen in young and middle-aged men, ${ }^{3}$ PAES can be a source of significant disability and impairment in affected patients. According to the Heidelberg classification system, PAES is classified into three types. Type I is characterized by an atypical course of the popliteal artery, type II occurs as a result of an abnormal muscular insertion, and type III exhibits both of these anatomic irregularities. ${ }^{4}$

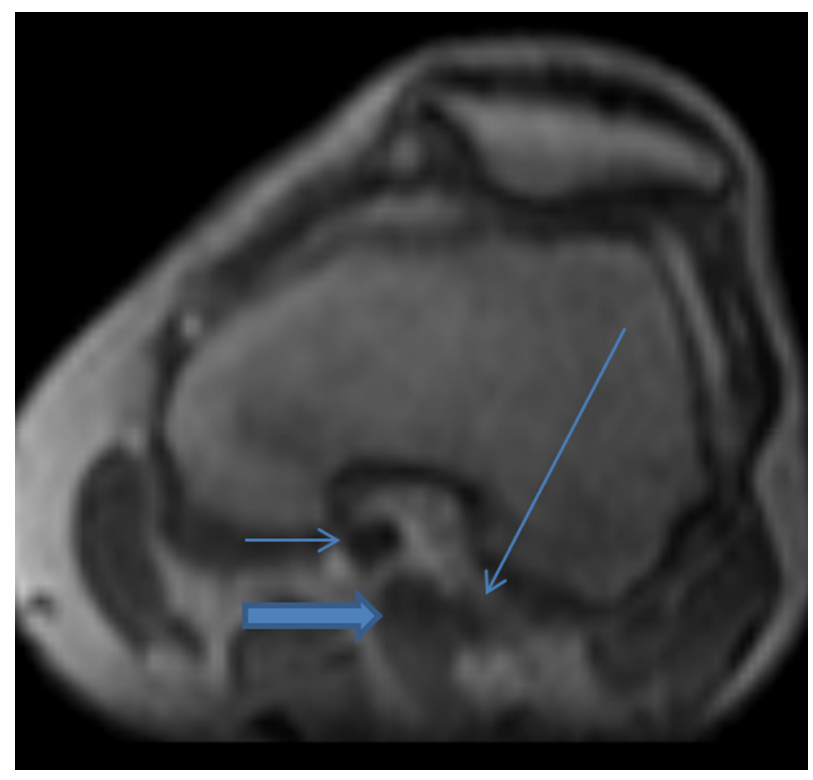

Figure 3 Reconstructed flash 3D post-contrast TI-weighted magnetic resonance image of the left knee in the transverse plane demonstrates the medial head of the gastrocnemius muscle (thick arrow) between the occluded popliteal artery (short thin arrow) and the popliteal vein (long thin arrow). 


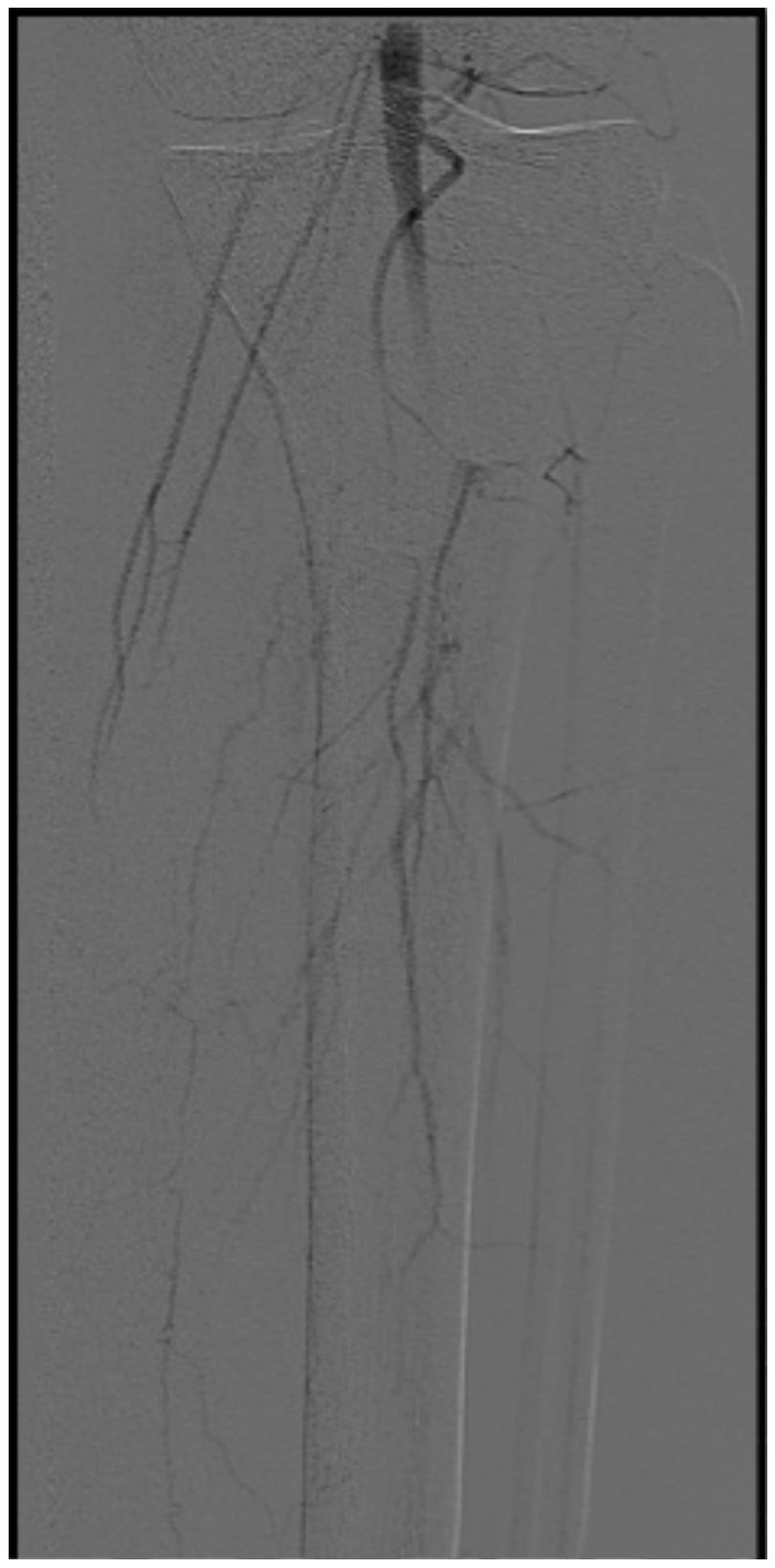

Figure $4 \mathrm{AP}$ image from digital subtraction left lower extremity arteriogram demonstrates thrombosis of the majority of the proximal runoff vessels.

Abbreviation: AP, anterior-posterior.

This abnormal anatomy creates extrinsic compression which can over time cause vascular compression that can lead to damage. ${ }^{5}$ Symptoms often occur as a result of arterial wall degeneration and compression, depending on the degree of compression, magnitude of forces acting on the popliteal artery, and duration of compression. ${ }^{6}$ If untreated, the compression mechanism frequently results in deterioration of the popliteal artery which may progress to an occlusion. ${ }^{7}$ Recurrent popliteal artery compression can cause intimal damage, thrombosis ${ }^{8}$ distal embolization, post-stenotic dilation, and formation of true aneurysm, ${ }^{6}$ which makes

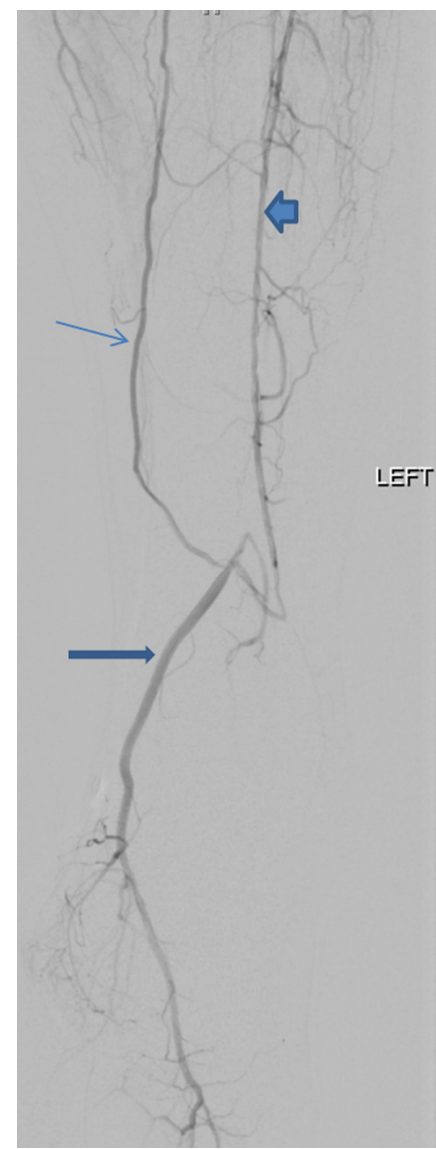

Figure 5 Lateral view from digital subtraction left lower extremity arteriogram demonstrates reconstitution of the posterior tibial artery (thick arrow) into the foot from a collateral vessel (thin arrow) and a diseased anterior tibial artery (block arrow).

accurate diagnosis and timely management imperative. ${ }^{7}$ Unfortunately, delayed diagnosis due to young patient age and lack of atherosclerotic risk factors is common, making progressive deterioration more frequent. The diagnosis is further complicated because PAES can be difficult to separate from other causes of lower limb pain. ${ }^{9}$ Clinical diagnosis is made with careful history, physical exam, imaging testing including ultrasonography, magnetic resonance angiography (MRA), computed tomography angiography (CTA), or catheter-based angiography. ${ }^{7}$ The most common presentation is intermittent claudication in young or middle-aged athletic patients without any context of atherosclerosis. ${ }^{4}$ Doppler ultrasound is a non-invasive modality that provides a good overview of the popliteal fossa, popliteal artery, and arterial flow at a reasonable cost. ${ }^{10}$ Magnetic resonance angiography and CTA allow physicians to visualize the popliteal fossa and arterial anomalies, providing better arterial depiction than ultrasonography with excellent visualization of stenosis, dilation, and arterial wall abnormalities. ${ }^{9}$ Furthermore, magnetic resonance angiography with high-contrast resolution offers precise analysis of muscle courses and their potential con- 


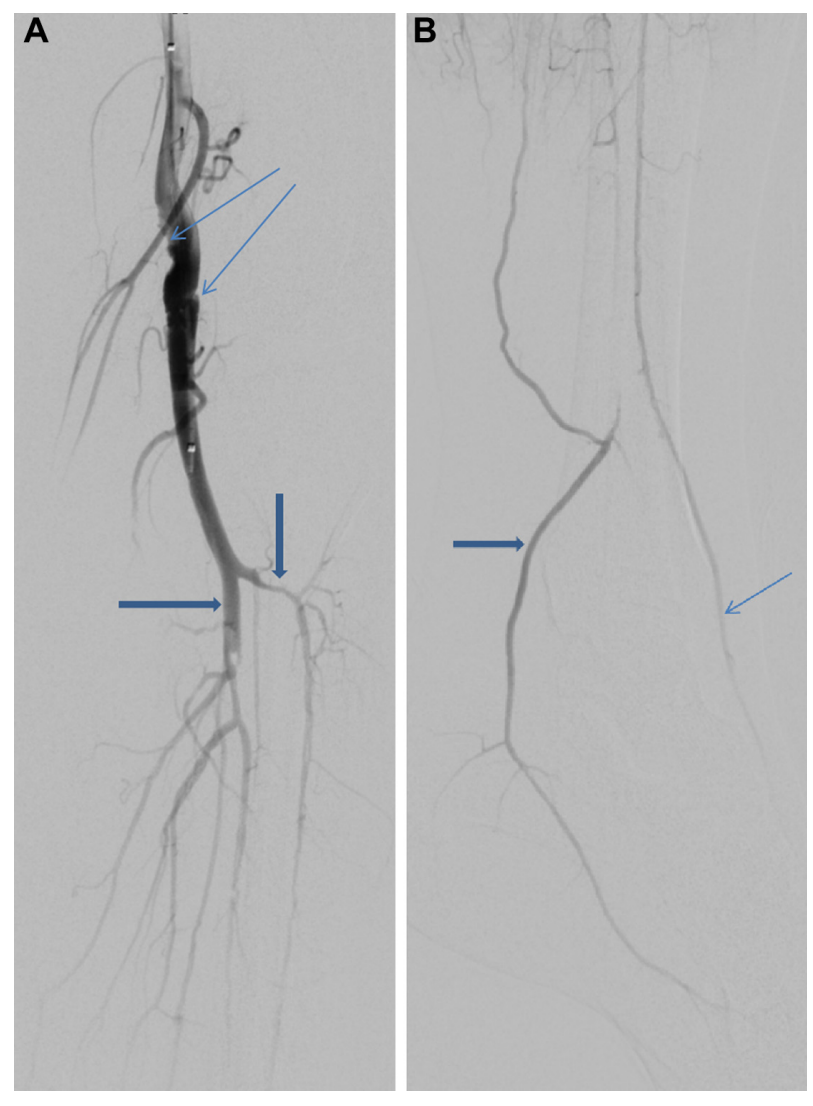

Figure 6 Digital subtraction angiographic (DSA) images following lytic therapy. Notes: (A) post-lytic therapy, anterior-posterior view from digital subtraction angiography (DSA) of left lower extremity demonstrates a persistent web and irregularity in the above-knee popliteal artery near the joint (thin arrows, marking the distal end of the previously seen clot in this location) and significant improvement of the thrombus in the tibioperoneal trunk (thick horizontal arrow) and diseased proximal runoff which is mainly collateral vessels and a diseased anterior tibial artery (thick vertical arrow). (B) Image from completion DSA of left lower extremity (lateral view) demonstrates improved filling of the posterior tibial artery (thick arrow) and dorsalis pedis artery (thin arrow, not filling prior to tPA) at the ankle via collaterals and a highly diseased anterior tibial artery into an intact pedal-plantar loop (not shown).

flict with popliteal vessels as well as allowing classification, making it ideal for diagnosis. ${ }^{11}$ However, CTA is less expensive and more available, making it a close second. Once the diagnosis of PAES has been made, several treatment options are available, with the treatment objective being to release the popliteal artery from compression and preserve popliteal arterial flow. ${ }^{5}$ Conventional surgery, endovascular surgery, thrombolysis, or a combination of these modalities are all reasonable treatment options depending on the patient's clinical symptomology and anatomy. ${ }^{9}$ This decision making can be very challenging. If the artery is occluded, stenotic, or aneurysmal, vascular reconstruction is mandatory in addition to the division of any entrapping structure. In patients with an acute occlusion, thrombolysis and angioplasty prior to surgery are appropriate, but have a high risk of reocclusion and will not be effective without removing the underlying reason for the popliteal artery entrapment. ${ }^{12}$
Our patient represents a typical case of PAES in that he is a middle-aged man presenting with acute progressive calf claudication. He was progressively symptomatic for 2 weeks before seeking treatment, resulting in a total occlusion. Diagnosis was confirmed using MRA and catheter-based angiography. Patient has irregularity of the infrapopliteal arteries mostly consistent with thromboembolic disease and less likely to represent Buerger's disease. This is based on the repeat catheter-based angiogram images showing improvement of the infrapopliteal arteries following lytic therapy. A diagnosis of Buerger's disease made via angiography is based on the presence of segmental occlusions and "corkscrew" collaterals around the area of the occlusion. ${ }^{13}$ As these observations were not made in the repeat angiography, it was concluded that the diagnosis of Buerger's disease was less likely. Options for treatment included release of compression and arterial repair versus bypass alone. The final decision was made for bypass alone since the patient's artery had been damaged over time, and since muscular release surgery is a significant surgery posing more risk to the patient.

Although other cases of PAES have been published, ${ }^{14,15}$ this case remains relevant due to the severity of presentation. The patient presented with complete occlusion of the left popliteal artery, which required multidimensional treatment. A combination of catheter-based lytic therapy, anticoagulation therapy, and surgical intervention was successfully implemented.

\section{Summary}

We present a challenging case of PAES with complicated decision making regarding an appropriate revascularization option with lowest risk.

We would recommend considering PAES in young patients with calf claudication. If untreated, the compression caused by this abnormal anatomy frequently results in damage to the popliteal artery which may give rise to an occlusion, ${ }^{8}$ making timely diagnosis and management essential. ${ }^{4}$

\section{Disclosure}

The authors have no conflicts of interest related to this work.

\section{References}

1. Insua JA, Young JR, Humphries AW. Popliteal artery entrapment syndrome. Arch Surg. 1970;101:771-775.

2. Igari K, Sugano N, Kudo T, et al. Surgical treatment for popliteal artery entrapment syndrome. Ann Vasc Dis. 2014;7(1):28-33.

3. Ellis DA, Williamson WK. Bilateral popliteal artery entrapment syndrome in a young man. J Vasc Surg. 2013;58(6):1669. 
4. Radonić V, Koplić S, Giunio L, Bozić I, Masković J, Buća A. Popliteal artery entrapment syndrome: diagnosis and management, with report of three cases. Tex Heart Inst J. 2000;27(1):3-13.

5. Halliday AW, Taylor PR, Wolfe JH, Mansfield AO. The management of popliteal aneurysm: the importance of early surgical repair. Ann R Coll Surg Engl. 1991;73:253-257.

6. López Garcia D, Arranz MA, Tagarro S, Camarero SR, Gonzalez ME, Gimeno MG. Bilateral popliteal aneurysm as a result of vascular type IV entrapment in a young patient: a report of an exceptional case. J Vasc Surg. 2007;46:1047-1050.

7. Simsek E, Burgra O, Teber MA, Katircioglu SF. What should be the first treatment of popliteal artery entrapment syndrome. Ann Thorac Cardiovasc Surg. 2014;20:169-172.

8. Iwai T, Konno S, Soga K, et al. Diagnostic and pathological considerations in the popliteal artery entrapment syndrome. J Cardiovasc Surg (Torino). 1983;24:243-249.

9. Lejay A, Ohana M, Lee JT, et al; Groupe Européen de Recherche sur les Prothèses Appliquées à la Chirurgie Vasculaire (GEPROVAS). Popliteal artery entrapment syndrome. J Cardiovasc Surg (Torino). 2014; 55(2 Suppl 1):225-237.
10. Altintas U, Helgstrand UV, Hansen MA, Stentzer KF, Schroeder TV, Eiberg JP. Popliteal artery entrapment syndrome: ultrasound imaging, intraoperative findings, and clinical outcome. Vasc Endovascular Surg. 2013;47:513-518.

11. Tercan F, Oğuzkurt L, Kizilkiliç O, Yeniocak A, Gülcan O. Popliteal artery entrapment syndrome. Diagn Interv Radiol. 2005;11:222-224.

12. di Marzo L, Cavallaro A, Sciacca V, Mingoli A, Tamburelli A Surgical treatment of popliteal artery entrapment syndrome: a ten-year experience. Eur J Vasc Surg. 1991;5:59-64.

13. Yoshimuta T, Akutsu K, Okajima T, Tamori Y, Kubota Y, Takeshita S. Corkscrew collaterals in Buerger's disease. Can J Cardiol. 2009;25(6):365.

14. Lee EJ, Jung JS, Lee K, Lee SH, Son HS, Sun K. Popliteal artery entrapment syndrome: a case with bilateral different types. Korean J Thorac Cardiovasc Surg. 2014;47:302-305.

15. Gemayel G, Murith N, Mugnai D, Khabiri E, Kalangos A. Popliteal artery entrapment syndrome: report of two cases. Vascular. 2012;20(6): 314-317.
International Journal of General Medicine

\section{Publish your work in this journal}

The International Journal of General Medicine is an international, peer-reviewed open-access journal that focuses on general and internal medicine, pathogenesis, epidemiology, diagnosis, monitoring and treatment protocols. The journal is characterized by the rapid reporting of reviews, original research and clinical studies across all disease areas.

\section{Dovepress}

A key focus is the elucidation of disease processes and management protocols resulting in improved outcomes for the patient. The manuscript management system is completely online and includes a very quick and fair peer-review system. Visit http://www.dovepress.com/ testimonials.php to read real quotes from published authors.

Submit your manuscript here: http://www.dovepress.com/international-journal-of-general-medicine-journal 